\title{
Characteristics of patients who visit the dental emergency room in a dental college hospital
}

\author{
Chihun $\mathrm{Kim}^{1 *}$, Eunhye Choi ${ }^{1 *}$, Kyeong-Mee Park', Eun-Jung Kwak ${ }^{2}$, Jisun Huh ${ }^{3}$, Wonse Park \\ 'Department of Advanced General Dentistry, College of Dentistry, Yonsei University, Seoul, South Korea \\ ${ }^{2}$ Seoul Dental Hospital for the Disabled, Seoul, Korea \\ ${ }^{3}$ Dental Education Research Center, College of Dentistry, Yonsei University, Seoul, South Korea
}

\begin{abstract}
Background: Emergencies in dentistry can be classified as medical and dental. Medical emergencies occur mainly during dental treatment in patients with a systemic disease. Dental emergency departments are largely divided into dental emergency rooms located in dental college hospitals and medical emergency rooms located in medical institutions. This study aimed to analyze the characteristics of and provide help to dental emergency patients in a dental hospital.

Methods: Overall, 1806 patients admitted to a dental emergency room at Yonsei University Dental Hospital for 1 year were included. The data collection period was from October 1, 2014 to September 30, 2015. An investigator reviewed medical records from the electronic medical record (EMR) system and radiographs. Results: The patients were 1,070 men and 736 women. The sex ratio was $1.45: 1$. The commonest age group was of 0-9 years, including $451(25.0 \%)$ patients, followed by $20-29$ years, including $353(19.5 \%)$ patients, and 30-39 years, including 277 (15.3\%) patients. Of the 108 patients transferred to the Severance emergency department, 81 had trauma, 19 were in pain, 4 were bleeding, and 4 had other complaints. Among chief complaints, 1,079 patients $(60.3 \%)$ had trauma, $564(31.5 \%)$ had pain, and $75(4.2 \%)$ had bleeding. Twenty-three cases $(1.3 \%)$ were caused by temporomandibular disorder (TMD).

Conclusion: Dentists should be able to adequately assess patients in a dental emergency room and treat trauma, pain, and bleeding.
\end{abstract}

Keywords: Bleeding; Dental Emergency; Infection; Pain; Retrospective Study.

This is an Open Access article distributed under the terms of the Creative Commons Attribution Non-Commercial License (http://creativecommons.org/licenses/by-nc/4.0/) which permits unrestricted non-commercial use, distribution, and reproduction in any medium, provided the original work is properly cited.

\section{INTRODUCTION}

Emergencies in dental hospitals can be broadly divided into medical and dental emergencies. Life-threatening medical emergencies usually occur during the dental treatment of patients with systemic diseases [1], while dental emergencies are associated with the dental procedures that involve trauma, pain, and bleeding.
Therefore, analyzing the characteristics that cause an emergency in patients helps better resolve their chief complaint by changing the treatment.

Depending on their timing and nature, dental emergencies can be divided into those that occur during the dental treatment, those that occur after the treatment, and those that are independent of the treatment. Most of the emergencies during the dental treatment are medical emergencies such as syncope, while emergencies after the

Received: December 19, 2018 - Revised: January 14, 2019 • Accepted: January 21, 2019

Corresponding Author: Wonse Park, Department of Advanced General Dentistry, College of Dentistry, Yonsei University, Yonseiro 50-1, Shinchondong, Seoul 03722, South Korea

Tel: +82-2-2228-8980 Fax: +82-2-2227-8906 E-mail: wonse@yuhs.ac

${ }^{*}$ Two authors (Kim and Choi) contributed equally to this study.

Copyright@ 2019 Journal of Dental Anesthesia and Pain Medicine 
treatment are associated with postoperative pain and bleeding having a clear cause. Emergencies independent of the treatment are mainly associated with traumas, such as dental, periodontal tissue, and jawbone traumas, and with acute dental pain including acute pulpitis and acute periodontitis caused by not receiving dental treatment in addition to the trauma [2]. When an emergency occurs in the morning or afternoon, patients can visit the nearest private dental clinic for outpatient treatment, but it is difficult to find dental emergency rooms in Korea in the evening and night when outpatient treatment is impossible. Therefore, it is very important to properly analyze the characteristics of patients visiting at night because without appropriate initial measures, patients may suffer pain or a delay in treatment, which leads to aftereffects on oral tissue functions such as the masticatory, articulative, and aesthetic functions [3-6].

The research on emergency patients who visit dental hospitals has been chiefly associated with the general emergency rooms of hospitals and has been concentrated on oral and maxillofacial surgery centered around maxillofacial trauma [7-13]. This study aimed to analyze the characteristics of dental emergency patients who visited dental hospital emergency rooms instead of general emergency rooms, particularly those who visited at night, to help with effective dental emergency care.

\section{MATERIALS AND METHODS}

In this study, the medical records of the 1806 patients who visited the dental emergency department of Yonsei University College of Dentistry in 1 year were reviewed. Among 1806 patients, 1070 men and 736 women were included. The data collection period was from October 1, 2014 to September 30, 2015. One investigator reviewed the medical records using EMR and radiographs. Patients were classified based on trauma, pain, bleeding, and other conditions, and deep infection was included as pain. Patients were classified according to the gender, age, and time, day, and month of visit.

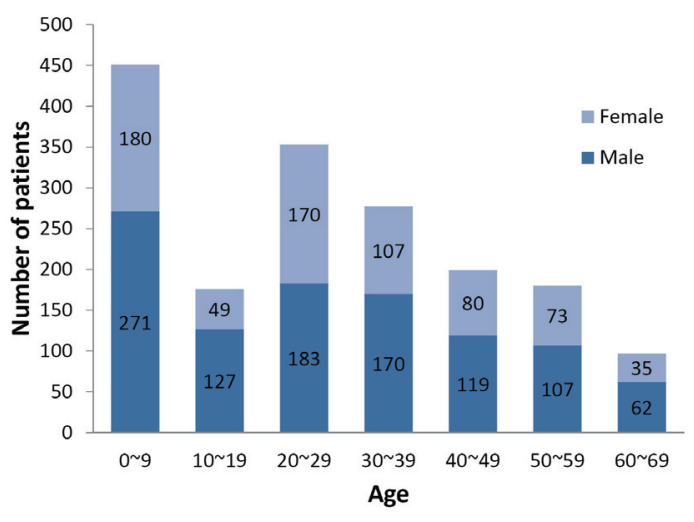

Fig. 1. Age distribution

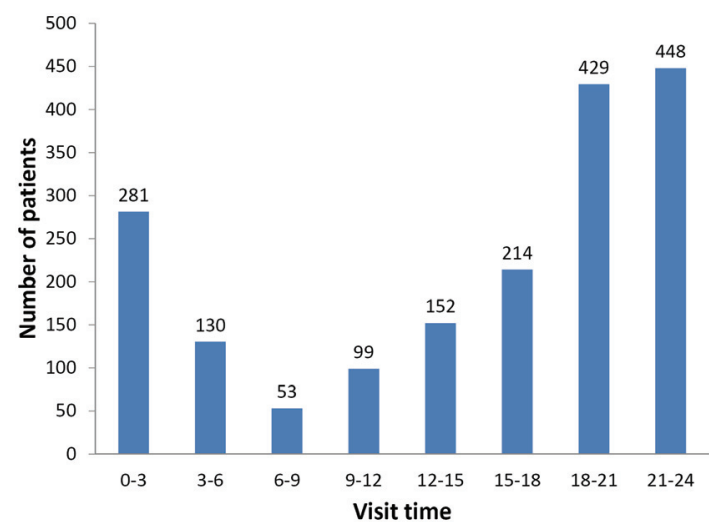

Fig. 2. Distribution according to visiting time

\section{RESULTS}

The patients were 1070 men and 736 women, resulting in a sex ratio of 1.45:1. The most common age range was 0-9 years (451 patients, $25.0 \%$ ), followed by $20-29$ years (353 patients, 19.5\%), and 30-39 years (277 patients, 15.3\%) (Fig. 1).

The most frequent time to visit the emergency room was between 21 and 24 hours (448 patients, 24.8\%), followed by the time between 18 and 21 hours (429 patients, 23.8\%), and between 0 and 3 hours (281 patients, 15.6\%) (Fig. 2).

Patients mostly reported in the month of May (215 patients, 11.9\%), followed by October (175 patients, 9.7\%), and September (174 patients, 9.6\%) (Fig. 3). The largest number of patients visited on Sunday (533 patients, $29.5 \%$ ), followed by Saturday (410 patients, 


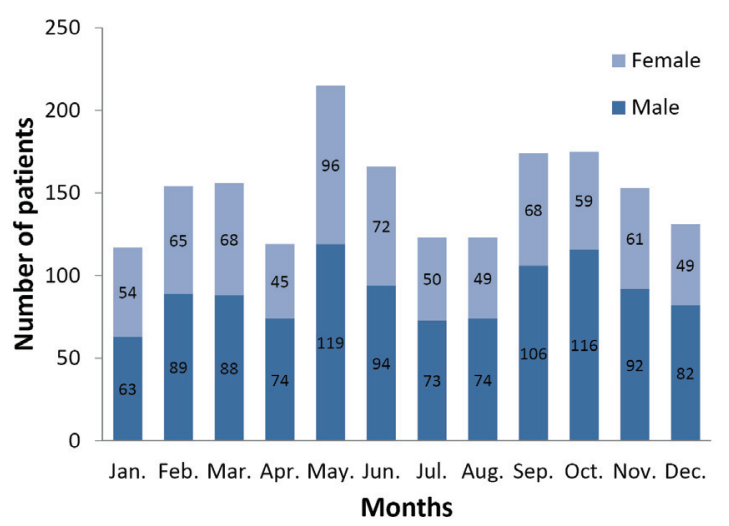

Fig. 3. Monthly incidence

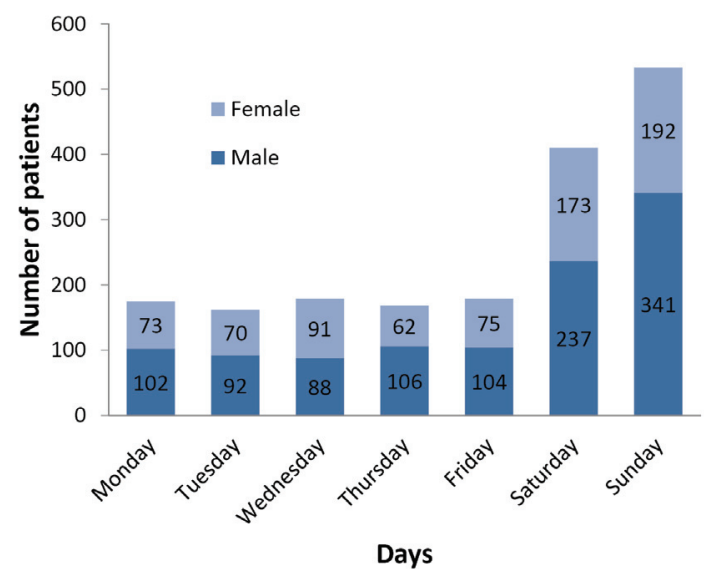

Fig. 4. Incidence by day

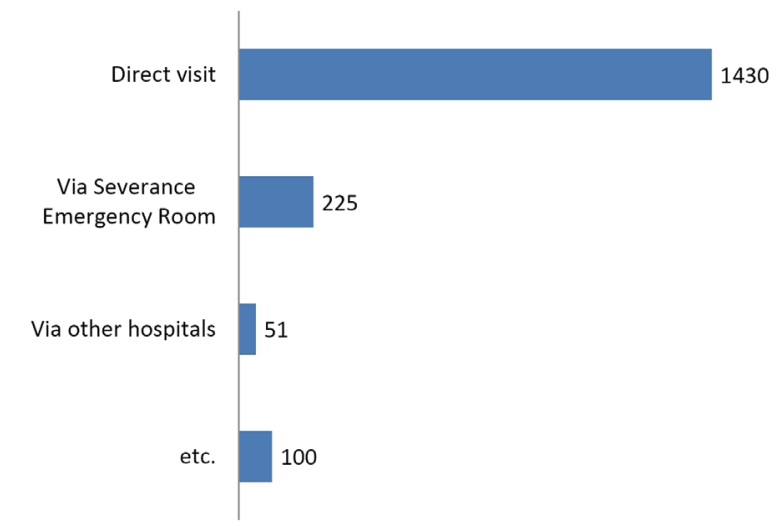

Fig. 5. Visit route

22.7\%). More than half the patients (52.2\%) visited in the weekend (Saturday and Sunday) (Fig. 4).

The most frequent route to the hospital was a direct visit to the dental emergency room by 1430 (78.5\%) patients, followed by Severance Emergency Room by 225

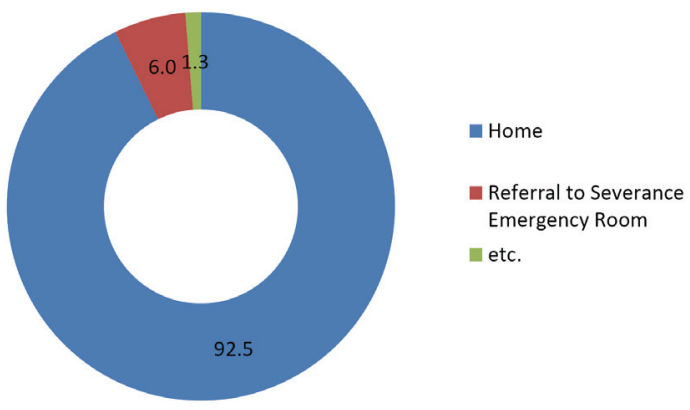

Fig. 6. Progress after emergency treatment

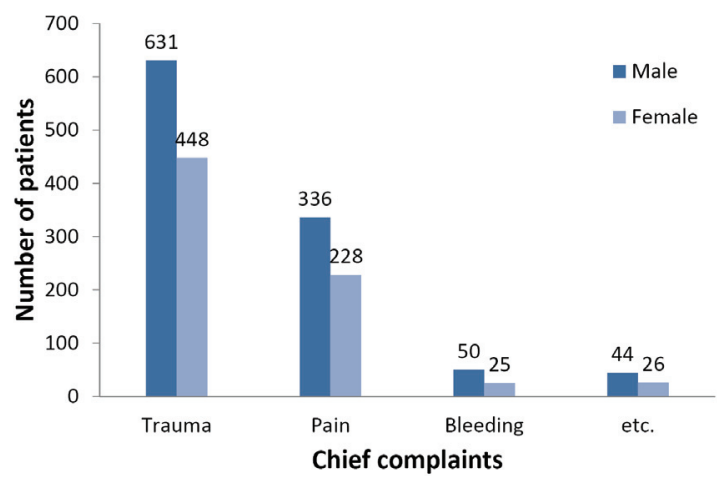

Fig. 7. Chief complaints

(12.5\%) patients, and the ambulance service by 100 (5.5\%) patients (Fig. 5).

After the treatment in the dental emergency room, 1671 (92.5\%) returned home and 108 (6.0\%) were transferred to the Severance Emergency Room. Of the 108 patients transferred to the Severance Emergency Room, 81 had trauma, 19 were in pain, 4 were bleeding, and so on. In the case of trauma patients, post-dental treatment was performed for additional systematic evaluation including CT. Under the age of 10 years, most of the cases required a sedative method for dental treatment. All 15 children under 10 years of age who were transferred to the Severance Emergency Room were sedated to treat trauma (Fig. 6).

Based on chief complaints, majority of the emergencies were due to trauma (1079 patients, 60.3\%), followed by pain (564 patients, 31.5\%), and bleeding (75 patients, 4.2\%) (Fig. 7). Twenty-three cases (1.3\%) were caused by TMD, and there were cases of prosthesis dropout. The rate of trauma was highest in patients aged under 10 years 


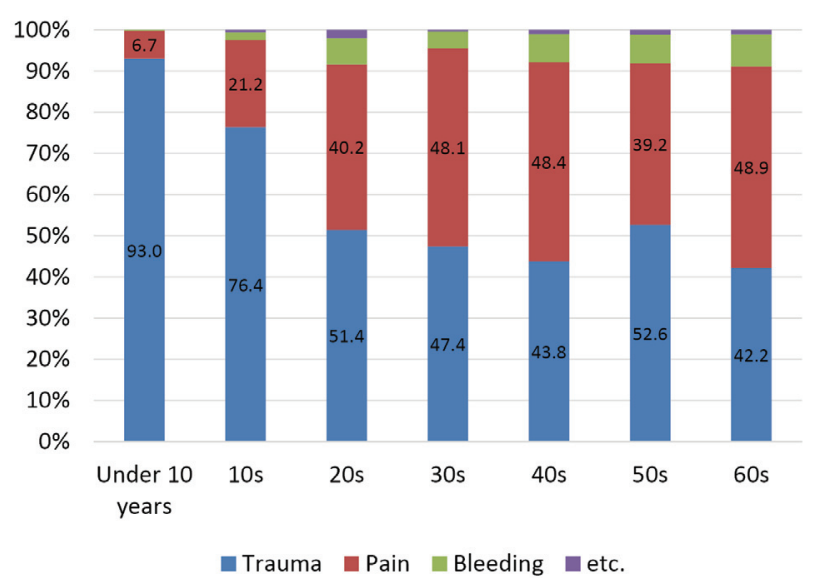

Fig. 8. Distribution according to the age and disease category

(414 patients, 93.0\%) and in teenagers (125 patients, $76.2 \%$ ), while the incidence of trauma and pain was similar in the 20s and older age groups (Fig. 8).

The average time to home and transfer was 1 hour 32 minutes after registration, and 830 patients $(46.0 \%)$ took mostly from 30 minutes to 1 hour. The treatment time for patients who had pain and trauma were 30 minutes to 1 hour, and 1-2 hours, respectively. Most of the patients who were referred because of bleeding were in the emergency room for more than 1 hour, which may be the time required for hemostasis (Fig. 9).

\section{DISCUSSION}

Most dental emergency rooms require the knowledge and skill for the diagnosis and emergency treatment of patients with trauma and various kinds of pain and bleeding as the chief complaint. Since the emergency room of Yonsei University Dental Hospital is operated independently of the Severance Emergency Room, they need the ability to determine whether to transfer the patients to the Severance Emergency Room.

The goal in dental emergencies is to conduct an accurate early diagnosis and perform emergency treatment accordingly, to reduce pain and possible complications, and in turn, improve the patient's quality of life [2]. Until now, most of the studies associated with dental emer-

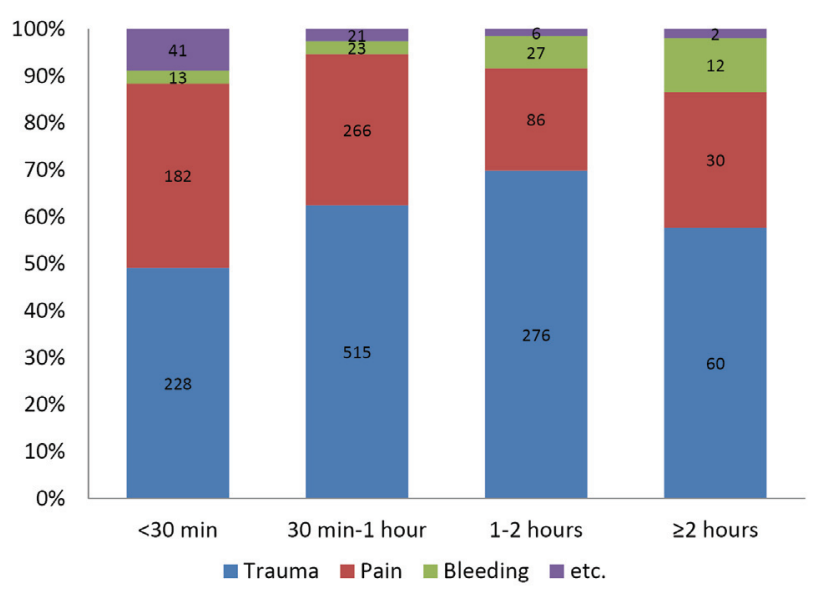

Fig. 9. Total time in the emergency room

gencies have been epidemiological surveys on all of the patients who visit the emergency room or has been centered around trauma patients, which is probably because the studies focused on emergencies in the field of oral and maxillofacial surgery. We performed this retrospective study because patients who visit dental hospitals at night may have different characteristics. According to the study of emergencies in dental college hospitals by You et al. in 2012 [14], out of 1,129 patients, $58.5 \%$ had trauma, $30.7 \%$ had pain, $8.5 \%$ had bleeding, and $2.3 \%$ had other characteristics, which is similar to this study, showing no regional characteristics. The total number of patients was 1,806 in this study, which is slightly more than the previous study, and it can be attributed to the regional demographic characteristics of Seoul and Cheonan.

Kim et al. (2012) [7] reported 58.7\% trauma, 21.9\% acute toothache and infection, and $12.7 \%$ bleeding patients. Jang et al. (2011) [8] reported 74.9\% trauma, $18.2 \%$ infection and toothache, and $6.3 \%$ bleeding patients, which has an overwhelming number of trauma patients. Keum et al. (2009) [15] reported 21.9\% trauma and $73.8 \%$ infection and toothache patients. The difference in the percentage of chief complaints according to the studies is considered to be because of the study of emergency rooms in general hospitals without a dental emergency room and the analysis of independent emergency rooms in dental hospitals. In other words, if 
the patient only has a dental injury, the patient will visit the dental emergency room, but if the patient has a dental trauma accompanied by jawbone or head and neck trauma, the patient will visit the medical emergency room. In addition, if the patient feels pain in the teeth, the patient believes that the pain can be controlled by visiting a dental emergency room rather than a general hospital's medical emergency room, so dental emergency rooms should be equipped with diagnostic equipment and treatment tools and materials to treat emergency patients with pain.

Overall, the successive order of age distribution of patients in this study was ages of under 10 years, $20 \mathrm{~s}$, and 30s; that of trauma patients was ages of under 10 years, 20s, and $30 \mathrm{~s}$, and that of pain patients was ages of $20 \mathrm{~s}, 30 \mathrm{~s}$, and $40 \mathrm{~s}$. In the study by You et al. [14], the order of patients was in their 20s, under 10 years old, and 40s, and in the case of trauma, the order of patients was under 10 years old, in their teens, and 20s, which accounted for $67.6 \%$ of the total number of trauma patients. Unlike trauma, patients with pain were in the order of $20 \mathrm{~s}, 40 \mathrm{~s}$, and $30 \mathrm{~s}$, so the age distribution of trauma and pain was different. The trend was similar in this study. In the case of trauma patients under 10 years old, parents only have 1-2 children because of the low fertility rate, and they take their children to the emergency room even in the case of minor trauma. Therefore, dentists in charge of dental emergency rooms should be familiar with dental trauma in the primary and mixed dentition. In particular, behavior control is difficult in terms of children with trauma, and since guardians can be sensitive and anxious, dentists should be well-trained for the behavioral control of the patient and guardian, their prognosis, and follow-up measures. If necessary, knowledge of drugs and emergency treatment is also required, as pediatric patients may need to be sedated through medication. There was no significant difference in the distribution by time period in all of the studies, as $20.5 \%$ of the patients visited between 9 pm-12 am and between 6 pm-9 pm, followed by $15.9 \%$ between 12 am-3 am, and there was no difference according to the trauma and pain group.

As for the monthly distribution of emergency patients, this study reported more emergency patients in May, September, and October, which was similar to other studies $[9,11,15,16]$. You et al. in 2012 [14] revealed unique results, as the number of emergency patients increased in March and September because there are five universities near Dankook University Dental Hospital hosting many events in the beginning of semesters. This study showed similar results, and in most of the studies, adult trauma patients increased in spring and fall, when social activities increase, and children trauma patients increased in spring and early summer, when outdoor activities increase. Patients with pain and infection increase when seasons change, which is considered to be because of the decrease in immunity during these periods.

According to the analysis of day of the week, patients visited more on weekends (Saturday and Sunday) rather than weekdays (Monday through Friday), when outpatient treatment is available. Patients visited more frequently on Sundays than Saturdays, which is considered to be because of more dental clinics opening on Saturday mornings or between 2 pm-4 pm in the afternoon. Most of the patients made direct visits, while 225 patients (12.5\%) were transferred from the Severance Hospital emergency room not knowing there was an emergency room in the dental hospital or because they were accompanied by other trauma. There were only a few prior studies on the follow-up after treatment in the dental emergency room, which seems to be because of the nature of the institution in which the dental and medical emergency rooms are divided, that is, $92.5 \%$ of the patients who visited the dental emergency room could return home after the treatment, but $6.0 \%$ needed extra care as they needed to be transferred to the medical emergency room. In other words, in the case of patients with multiple trauma, such as fracture of the jaw or trauma to other areas, patients with space infection who need to be hospitalized and those who need medication control because of bleeding caused by medication, a cooperative medical treatment system is needed between 
dental and medical emergency rooms because these patients cannot be treated by the dental emergency room alone. In case of children, behavioral control is important during emergency treatment, and if sedation is necessary, the medical emergency room needs to monitor the patient because it is difficult to perform sedation in the night-time emergency room. Therefore, it is important for dentists working in the dental emergency rooms to understand these cases and to develop the ability to transfer them quickly to the medical emergency room if necessary.

In this study, a total of 1,079 patients $(60.3 \%)$ had trauma, but their detailed assessment could not be performed. In the study by You et al. [14], the order was subluxation (16.48\%), laceration $(13.70 \%)$, and crown fracture without pulp exposure (11.95\%). Keum et al. (2009 [15]) reported the order was crown fracture, subluxation, concussion, avulsion, and alveolar bone fracture. Kim et al. (2001) [9] reported the order was avulsion, tooth fracture, tooth loss, and alveolar bone fracture. In the case of trauma, it is difficult to compare the study results, as they may differ in terms of patient perspective and odontology, since soft tissue and alveolar bone damage are frequently accompanied by multiple tooth damages. It is important to evaluate the prognosis after emergency treatment, and further research is required to examine the characteristics of trauma patients when they visit for treatment and the changes in the follow-up process after trauma. Detailed information on emergency patients associated with pain and bleeding should also be analyzed through further research.

The patients who visit dental emergency rooms are characterized trauma, pain, and bleeding, in that order. Dentists in dental emergency rooms should properly evaluate the patients to differentiate bleeding associated with extensive trauma, head and neck infections, and systemic diseases, including jawbone fractures that should be referred to the medical emergency room and should develop skills for emergency treatment associated with dental pulp, periodontal, and temporomandibular dislocations. Further studies on trauma, pain, and bleeding are necessary to determine the correct treatment and prognosis of patients who visit the dental emergency room.

\section{AUHHOR ORCIDS}

Chihun Kim: https://orcid.org/0000-0002-6163-0976

Eunhye Choi: http://orcid.org/0000-0003-2671-4370

Kyeong-Mee Park: http://orcid.org/0000-0002-1301-0959

Eun-Jung Kwak: http://orcid.org/0000-0001-5750-1170

Jisun Huh: https://orcid.org/0000-0001-5514-187X

Wonse Park: http://orcid.org/0000-0002-2081-1156

\section{CONFLICT OF INTEREST AND SOURCE OF FUNDING: This} research was supported by the Basic Science Research Program through the National Research Foundation of Korea (NRF) funded by the Ministry of Education (grant no. 2017R1D1A1B03033657).

\section{REFERENCES}

1. Little JW, Falace DA, Miller CS, et al. Dental management of the medically compromised patient. 5th ed. St Louis, Mosby. 1997.

2. Conley JJ. Complications of head \& neck surgery. 2nd ed. Philadelphia, Saunders. 1979.

3. Galea H. An investigation of dental injuries treated in an acute care general hospital. J Am Dent Assoc 1984; 109: 434-8.

4. Kaban LB, Pogrel MA, Perrott DH. Complications in oral and maxillofacial surgery. Philadelphia, Saunders. 1997.

5. Malamed SF, Robbins KS. Medical emergencies in dental office. 3rd ed. St Louis, Mosby. 1993.

6. McCarthy FM. Medical emergencies in dentistry. 3rd ed. Philadelphia, Saunders. 1982.

7. Kim SH, Kim DY, Baek JS, Jung TY, Park SJ. Characteristics of dental emergency patients at Busan Paik Hospital. J Korean Assoc Maxillofac Plast Reconstr Surg 2012; 34: 58-64.

8. Jang CS, Lee CY, Kim JW, Yim JH, Kim JY, Kim YH, et al. A clinical study on the dental emergency patients visiting an University Hospital emergency room. J Korean Assoc Oral Maxillofac Surg 2011; 37: 439-47. 
9. Kim JR, Chung IK, Yang DK, Park BW. A clinical study on the emergency patients of oral and maxillofacial surgery during recent 5 years. Maxillofac Plast Reconstr Surg 2001; 23: $155-62$.

10. Moon WK, Jung YS, Lee EW, Kwon HK, Yoo JH. The characteristics on the dental emergency patients of Wonju Christian Hospital for last 10 years. J Korean Assoc Oral Maxillofac Surg 2004; 30: 34-42.

11. Cho KS, Kim KY, Lee SH, Park HJ, So KS, Cho YK, et al. A clinical study on oral \& maxillofacial patients visiting Chonnam Univ-Hospital emergency room, J Korean Assoc Maxillofac Plast Reconstr Surg 1997; 19: 435-46.

12. Lee DK, Min SK, Yang CY, Mun C, Kim JG. A clinical study on the dental emergency patients visiting Wonkwang Univ-Hospital emergency room. J Korean Assoc Maxillofac Plast Reconstr Surg 2002; 24: 31-9.

13. Baik JS, Yoon KH, Park KS, Cheong JK, Shin JM, Choi
$\mathrm{MH}$, et al. A clinical study on the emergency patients of oral and maxillofacial surgery visiting Sang-Gye Paik Hospital emergency room. J Korean Assoc Maxillofac Plast Reconstr Surg 2008; 30: 561-6.

14. You TM, Park GJ. The clinical analysis on the patients visiting the dental emergency room. Kor J Oral Maxillofac Pathol 2012; 36: 323-30.

15. Keum KC, Paeng JY, Choi BY, Choi JG, Oh SR, Lee J, et al. An clinical analysis on the dental emergency patients visiting the emergency room of dental hospital of Won-Kwang University. Maxillofac Plast Reconstr Surg 2009; 31: 35-40.

16. Shim HG, Lee EW. A clinical study on nighttime emergency patients of the dental hospital Yonsei Medical Center. J Kor Assoc Oral Maxillofacial Surg 1985; 11: 259-70. 